\title{
Avaliação da eficiência técnica dos hospitais de ensino do Brasil utilizando a análise envoltória de dados
}

\author{
Assessing the technical efficiency of Brazil's teaching hospitals using \\ data envelopment analysis
}

Aline Garmatz (https://orcid.org/0000-0002-7561-925X) ${ }^{1}$

Guilherme Bergmann Borges Vieira (https://orcid.org/0000-0001-6463-9987) ${ }^{2}$

Sérgio Antonio Sirena (https://orcid.org/0000-0001-7267-546X) ${ }^{3}$

${ }^{1}$ Prefeitura Municipal de Porto Alegre. Praça Montevideo 10, Centro Histórico. 90010-170. Porto Alegre RS Brasil. garmatz@hotmail.com ${ }^{2}$ Universidade de Caxias do Sul. Caxias do Sul RS Brasil. ${ }^{3}$ Grupo Hospitalar Conceição. Porto Alegre RS Brasil.

\begin{abstract}
The general objective of this study was to assess the technical efficiency of Brazil's teaching hospitals using data envelopment analysis (DEA). To this end, a quantitative exploratory study was conducted with secondary data from the national health information system (DATASUS) using an output-oriented DEA model. The study population consisted of 29 large-sized teaching hospitals located in the country's Center-West, South, Southeast, and North regions. Twelve hospitals were shown to be on the efficient frontier (technically efficient) and 17 were off the frontier. Absolute efficiency values were calculated for the hospitals that were off the frontier, using benchmarks with weighting for benchmarking. Private for-profit hospitals were shown to be the most efficient, followed by private not-for-profit and public facilities. The findings of this study suggest that DEA has potential for assessing technical efficiency in hospital settings in relation to operational capacity.
\end{abstract}

Key words Teaching hospitals, Unified Health System, Health service evaluation, Benchmarking, Operations research
Resumo O objetivo geral deste estudo foi avaliar a eficiência técnica dos hospitais de ensino do Brasil por meio da análise envoltória de dados. Para tanto, foi realizada uma pesquisa exploratória de natureza quantitativa utilizando dados secundários do Ministério da Saúde. Foi utilizado o método da análise envoltória de dados (DEA - Data Envelopment Analysis), orientada a output. A população deste estudo constituiu em 29 hospitais de ensino de grande porte nas regiões Centro-Oeste, Sul, Sudeste e Norte do Brasil. Identificou-se que 12 hospitais ficaram localizados na fronteira de eficiência, ou seja, foram relacionados como eficientes na maximização dos outputs (produtos), $e$ 17 ficaram abaixo. Foi estabelecido o valor absoluto para a eficiência daqueles que não atingiram a fronteira por meio de benchmarks com pesos para o espelhamento. Identificou-se, quanto à natureza jurídica, que a personalidade "entidades empresariais" foi mais eficiente, seguida das "entidades sem fins lucrativos" e, por último, da "administração pública". Os resultados desta pesquisa sugerem que a DEA tem potencial para avaliação de eficiência técnica no âmbito hospitalar quando avaliada a capacidade de produção do estabelecimento. Palavras-chave Hospitais de ensino, Sistema Único de Saúde, Avaliação em saúde, Benchmarking, Pesquisa operacional 


\section{Introdução}

Ao longo dos últimos anos, os gestores no Brasil têm pesquisado alternativas para a otimização dos recursos físicos e financeiros, buscando a excelência da qualidade do atendimento na área da saúde. A crise financeira que atinge o Brasil, com uma grave repercussão na saúde pública, gera a necessidade da busca de soluções para a redução de custos, a fim de viabilizar o aumento da produção com qualidade e a ampliação do acesso da população aos serviços de saúde.

A universalização da saúde, garantida pela Constituição de 1988, trouxe para a população brasileira a esperança de que, sempre que preciso, suas demandas de saúde estariam garantidas e protegidas pelo Estado brasileiro. No entanto, percebe-se que manter um sistema de saúde aberto a todos é extremamente complexo, por inúmeros motivos. A escassez de recursos financeiros e a ausência de uma estrutura física capaz de atender toda a população de uma região são alguns desses motivos que tornam a gerência dos estabelecimentos de saúde um grande desafio ${ }^{1}$.

Gerir uma organização de saúde, qualquer que seja o seu porte, em um sistema complexo requer capacitação, desenvolvimento de competências, inovação constante e postura empreendedora. Na briga pela competitividade, urge a necessidade de agregar valor à produção, por meio da criação de diferenciais, obtidos à custa de inovação de produtos e processos. Esse novo modelo de produzir, além de exigir profissionalização da gestão, requer dos colaboradores flexibilidade e iniciativa para a realização de inúmeras tarefas simultâneas e complexas, além da resolução de problemas inesperados ${ }^{2}$.

As dificuldades que envolvem o setor de saúde no Brasil são muitas. Destacam-se, ao analisar os estabelecimentos hospitalares, as políticas setoriais e o modelo de saúde que vem sendo adotado nas últimas décadas. Torna-se necessário, então, racionalizar as ações em saúde, analisando as relações de custo-efetividade, custo-benefício e minimizar os equívocos no direcionamento dos investimentos e na condução das políticas públicas voltadas ao setor, buscando sempre a otimização dos recursos escassos ${ }^{3}$.

Os hospitais de ensino possuem os recursos de saúde mais complexos do SUS, nos quais foram realizados volumosos investimentos públicos e que realizam os procedimentos mais custosos ao sistema. Eles provêm serviços imprescindíveis para o desenvolvimento da saúde da população, por meio da pesquisa, do ensino e da assistência. Além disso, esses hospitais precisam atentar para seu importante papel na viabilização dos princípios do SUS, em especial de sua diretriz de integralidade. $\mathrm{O}$ acesso aos seus serviços deve estar integrado às demais unidades de saúde do sistema e colaborar para que todos os pacientes consigam ingresso ao que há de mais moderno e útil para melhorar a sua saúde ${ }^{4}$.

$\mathrm{Na}$ área hospitalar, estudos de avaliação do desempenho produtivo exigem a constituição de modelos, que descrevem de forma abstrata, simplificada e sistematizada as características dessas organizações, a fim de que elas sejam compreendidas sob o prisma de interesse. Modelos são representações parciais da realidade, construídas para um propósito definido. Todavia, devem ser idealizados a partir de uma teoria, suficientemente abrangente, de forma que reflitam bem a realidade, que seus resultados sejam consistentes com a teoria subjacente e que possam ser aplicados ao propósito desejado. Diferentes teorias têm fundamentado a construção de modelos de hospitais tendo em vista a heterogeneidade de propósitos e os diferentes enfoques e perspectivas dos estudos. Por outro lado, as características específicas dos hospitais e de seus serviços de assistência à saúde têm limitado, e também dificultado, a realização de estudos comparativos ${ }^{5}$.

O mundo globalizado estimulou mudanças no processo de transformação em busca da sustentabilidade. Organizações atuantes nos diferentes setores, destacando o da saúde, tiveram que mudar para sobreviver, assim os ditames da competitividade passaram a ser delimitados pelas variáveis agilidade, produtividade e qualidade, ou seja, por critérios de eficiência e eficácia dos processos produtivos. No que se refere aos sistemas de produção, existe o pensamento lean (enxuto, originário do sistema Toyota de produção), que traz inúmeros exemplos de boas práticas. Conceitos de produção enxuta aplicada aos sistemas de produção são diretamente influentes sobre planejamento, programação, controle da produção e, consequentemente, sobre o desempenho operacional da organização. A abordagem lean está inteiramente relacionada à abolição de desperdícios. Esse sistema de produção surgiu como um sistema de manufatura cujo objetivo é aperfeiçoar os processos e operações por meio da diminuição contínua de desperdícios². De modo geral, a preocupação com a eficiência chega a ser criticada, como um estímulo ao enxugamento de serviços, mas quando a eficiência é critério de avaliação da gestão, faz sentido buscar métodos gerenciais. A apuração de indicadores não é pro- 
blema intransponível tecnicamente, mas sua implantação depende de política institucional e se justifica quando serve para tomar decisões. Caso contrário, termina por ser desculpa para processos pontuais, orientados por agentes externos ao setor com pouco conhecimento sobre as especificidades de seus processos ${ }^{6}$.

Um estudo internacional destaca que em algum momento o hospital irá além do seu nível ideal de eficiência e começará a mostrar deseconomias de escala. O ideal é encontrado quando todas as economias de escala foram exploradas, mas ainda sem criar deseconomias. Gestores de hospitais e formuladores de políticas devem se concentrar no uso eficiente de insumos para a produção de serviços de saúde e estar cientes de que é possível reduzir as entradas sem comprometer os serviços e cuidados prestados aos pacientes. Compreender os fatores-chave que levam à ineficiência permite aos hospitais melhorar a saída sem buscar recursos adicionais ou manter o volume de recursos oferecidos diminuindo os excessos nas entradas ${ }^{7}$.

Um dos desafios para avaliar a gestão em saúde - de natureza determinística, em vez de probabilística - é encontrar técnicas que permitam analisar concomitantemente todos os aspectos envolvidos. A análise envoltória de dados (DEA, do inglês data envelopment analysis) é uma metodologia vastamente empregada no estudo de produtividade e eficiência técnica de organizações que empregam múltiplos insumos para gerar múltiplos produtos. Admite identificar as melhores práticas por meio de fronteiras empíricas de programação linear ${ }^{8}$.

A DEA foi desenvolvida por A. Charnes, W.W. Cooper e E. Rhodes para avaliar organizações sem fins lucrativos e do setor público. Posteriormente, a técnica encontrou aplicações em organizações com fins lucrativos. A DEA compara cada unidade de prestação dos serviços a todas as outras unidades de uma organização em múltiplos locais, além de calcular uma classificação de eficiência baseada nos índices de entrada e de saída de recursos. Inúmeros dados de entrada (por exemplo, horas de trabalho e materiais) e de saída (por exemplo, vendas e indicações) são possíveis e desejáveis ao medir a eficiência de uma unidade. Assim, o modelo de programação linear define a margem de eficiência com base naquelas poucas unidades que produzem com uma eficiência de $100 \%$ (melhor resultado da amostra). As áreas que precisam de melhorias são identificadas ao comparar as práticas operacionais das unidades eficientes àquelas das unidades menos eficientes. O compartilhamento das práticas de gerenciamento das unidades mais eficientes com as menos eficientes possibilita uma oportunidade para a melhoria dessas unidades e o incremento da produtividade do sistema total ${ }^{9}$.

A DEA consiste em um método não paramétrico, ou seja, não estabelece uma forma funcional explícita, relacionando as variáveis para mensuração comparativa da eficiência de unidades tomadoras de decisão (decision making units - DMUs) com base nas melhores práticas. Essa técnica permite analisar a eficiência relativa de unidades produtivas com múltiplos insumos (inputs) e múltiplos produtos (outputs), intermediadas pela construção de uma fronteira virtual de eficiência e da identificação de ineficiências geradas por decisões e ações menos adequadas ${ }^{10}$.

A DEA procura elevar ao máximo a eficiência de uma unidade de serviço, expressa como a razão entre saídas e entradas, pela comparação da eficiência de uma dada unidade ao desempenho de um grupo de unidades similares que estão desempenhando o mesmo trabalho. No processo, algumas unidades atingem $100 \%$ de eficiência e são referidas como unidades eficientes, enquanto outras unidades, com índices de eficiência menores do que $100 \%$, são referidas como unidades ineficientes?.

Ao determinar as DMUs com as melhores práticas, a DEA constrói uma fronteira de produção empírica, e o grau de eficiência varia de 0,0 a 1,0 (ou de 0 a 100\%), dependendo da distância da unidade à fronteira. Para que as unidades sejam avaliadas como eficientes, aplica-se ainda a lógica de Pareto-Koopmans, na qual uma unidade presente na fronteira só será eficiente se não for possível reduzir nenhum input, ou aumentar qualquer output, sem que se tenha que aumentar simultaneamente outro input, ou reduzir outro output ${ }^{11}$.

As variáveis necessárias para o cálculo da eficiência relativa das DMUs são classificadas em inputs (entradas/insumos do sistema) e outputs (saídas/produtos do sistema). A eficiência relativa de cada DMU é determinada como a razão da soma ponderada de seus produtos (outputs) pela soma ponderada dos insumos necessários para gerá-los (inputs). Na DEA, essas variáveis são ajustadas por pesos, calculadas livremente ou de forma restrita por meio de programação linear, objetivando maximizar a eficiência de cada DMU em relação ao conjunto de referência ${ }^{12}$.

Quanto aos modelos de DEA, existem dois que são mais largamente utilizados: CCR e BCC. $\mathrm{O}$ primeiro tem sua sigla oriunda do nome dos 
autores Charnes, Cooper e Rhodes (1978), que publicaram o primeiro artigo sobre a DEA. O segundo é uma extensão do primeiro e tem sua sigla oriunda do nome dos autores Banker, Charnes e Cooper (1984) ${ }^{13}$. A distinção entre esses modelos é que o CCR assume a hipótese de retornos constantes de escala (também chamado de CRS - constant returns to scale) e o BCC considera retornos variáveis de escala (também chamado de VRS variable returns to scale). Os dois modelos possibilitam verificar se a produtividade pode ser aumentada com redução de insumos ou com aumento de produção. Além disso, a comparação entre os resultados dos modelos CCR e BCC admite identificar problemas decorrentes de alterações na escala de produção ou de ordem gerencial ${ }^{14}$.

Dessa forma, o presente estudo analisou a eficiência de produção dos hospitais de ensino do Brasil, evidenciando carências, potencialidades e indicando melhores formas para a racionalização dos recursos existentes a partir da seguinte pergunta de pesquisa: qual é o escore de eficiência técnica (hospitalar) dos hospitais gerais de ensino do Brasil a partir da análise envoltória de dados? A partir disso, foi proposto avaliar a eficiência técnica hospitalar dos hospitais de ensino do Brasil por meio da análise envoltória de dados, com os seguintes objetivos específicos: identificar os hospitais de ensino do Brasil eficientes tecnicamente segundo a abordagem da análise envoltória de dados, no âmbito de produção hospitalar; propor metas de produção hospitalar para os hospitais identificados como ineficientes tecnicamente; e comparar a eficiência técnica hospitalar dos hospitais de ensino do Brasil quanto à sua natureza jurídica.

\section{Material e métodos}

Trata-se de um estudo exploratório de natureza quantitativa aplicado na base de dados secundários de domínio público de todos os hospitais de ensino do Brasil, disponibilizados pelo Ministério da Saúde por meio do Departamento de Informática do SUS (DATASUS) em maio de 2017. Foram incluídos todos os hospitais que atenderam aos critérios de inclusão descritos adiante (com justificativa), a partir dos dados informados no Cadastro Nacional dos Estabelecimentos de Saúde (CNES). A população de hospitais foi intencional e não probabilística, com o objetivo de tornar a amostra homogênea e comparável, como estabelece a metodologia da análise envoltória de dados.
Ser hospital de ensino e geral (não especializado): hospitais especializados possuem atendimento em uma única especialidade, não possibilitando ser comparável, uma vez que essas especialidades podem ser distintas e com características muito diversificadas.

Ter mais de 150 leitos no total (hospitais de porte grande e especial): possuir porte semelhante.

Possuir leitos de Unidade de Tratamento Intensivo (UTI) adultos, pediátricos e neonatais: buscou equiparar os hospitais quanto à complexidade em todas as faixas etárias de atendimento.

Possuir leitos obstétricos e psiquiátricos/saúde mental: considerou a permanência mais baixa ocasionada por um parto quanto à longa permanência de um tratamento psiquiátrico/saúde mental.

Como diferencial, propôs-se nesse artigo um critério rígido de inclusão, principalmente no quesito capacidade instalada e tipologia. Também corrigiu limitações identificadas na literatura, como utilizar o número de horas em vez de número de profissionais, promovendo assim uma leitura mais próxima da realidade. Especificamente, em nível nacional existem poucos estudos na literatura que apontem para a eficiência dos hospitais de ensino (caracterização obtida por meio do CNES), não meramente os universitários vinculados à Empresa Brasileira de Serviços Hospitalares (EBSERH).

Essa metodologia é diretamente aplicável à utilização da técnica de DEA, em que os inputs representam os insumos e os outputs, os produtos. Na aplicação desse modelo, pode-se escolher entre duas opções: orientado ao output (obter o máximo nível de resultado mantendo os mesmos insumos) ou orientado ao input (obter o mínimo emprego de insumos mantendo o mesmo resultado $)^{15}$. Na maximização dos outputs dos dados de valor total faturado, número de AIHs, dias de permanência e procedimentos de alta complexidade, essa aplicação da DEA foi orientada para verificar quem gerou mais produtos com base nos insumos existentes, considerando uma escala de ganho constante (CCR). A escolha por essa orientação se deu principalmente pela incapacidade de o gestor público, nos níveis municipais e estaduais, ter gerência sobre questões internas de cada hospital contratualizado, e também pela escassez de recursos e alta demanda de usuários do SUS por atendimento no nível terciário. O Quadro 1 apresenta as variáveis que foram utilizadas para a aplicação da metodologia DEA e a fonte de dados utilizada para cada uma delas. 
Quadro 1. Varáveis utilizadas nesta pesquisa e em estudos correlatos.

\begin{tabular}{|c|c|c|c|c|}
\hline Orientação & Variáveis (total/ano) & $\begin{array}{c}\text { Identificação da } \\
\text { variável }\end{array}$ & Fonte dos dados & Estudos correlatos \\
\hline \multirow[t]{4}{*}{$\begin{array}{l}\text { Input } \\
\text { (insumos) }\end{array}$} & $\begin{array}{l}\text { Número total de } \\
\text { leitos SUS }\end{array}$ & LEITOS & $\begin{array}{l}\text { Cadastro Nacional de } \\
\text { Estabelecimentos de } \\
\text { Saúde-CNES }{ }^{16}\end{array}$ & $\begin{array}{l}\text { - Souza et al. }(2016)^{13} \\
\text { - Silva et al. }(2017)^{17} \\
\text { - Silva et al. }(2016)^{18}\end{array}$ \\
\hline & $\begin{array}{l}\text { Número total de } \\
\text { horas hospitalares } \\
\text { médicas SUS }\end{array}$ & HORAS MED & $\begin{array}{l}\text { Cadastro Nacional de } \\
\text { Estabelecimentos de } \\
\text { Saúde-CNES }{ }^{16}\end{array}$ & $\begin{array}{l}\text { - Souza et al. }(2016)^{13} \\
\text { - Lins et al. }(2007)^{19} \\
\text { - Silva et al. }(2017)^{17} \\
\text { - Silva et al. }(2016)^{18}\end{array}$ \\
\hline & $\begin{array}{l}\text { Número total de } \\
\text { horas hospitalares de } \\
\text { enfermeiros SUS }\end{array}$ & HORAS ENF SUP & $\begin{array}{l}\text { Cadastro Nacional de } \\
\text { Estabelecimentos de } \\
\text { Saúde }- \text { CNES }^{16} \\
\end{array}$ & $\begin{array}{l}\text { - Souza et al. }(2016)^{13} \\
\text { - Silva et al. }(2017)^{17} \\
\text { - Silva et al. }(2016)^{18}\end{array}$ \\
\hline & $\begin{array}{l}\text { Número total de } \\
\text { horas hospitalares de } \\
\text { enfermagem de nível } \\
\text { médio SUS } \\
\end{array}$ & $\begin{array}{l}\text { HORAS ENF } \\
\text { MED }\end{array}$ & $\begin{array}{l}\text { Cadastro Nacional de } \\
\text { Estabelecimentos de } \\
\text { Saúde - CNES }{ }^{16}\end{array}$ & $\begin{array}{l}\text { - Souza et al. }(2016)^{13} \\
\text { - Lins et al. }(2007)^{19} \\
\text { - Silva et al. }(2016)^{18}\end{array}$ \\
\hline \multirow[t]{4}{*}{$\begin{array}{l}\text { Output } \\
\text { (produtos) }\end{array}$} & $\begin{array}{l}\text { Valor total recebido } \\
\text { no faturamento } \\
\text { hospitalar SUS }\end{array}$ & VALOR & \begin{tabular}{|l|} 
Sistema de \\
Informação Hospitalar \\
Descentralizado - \\
SIHD $^{20}$ \\
\end{tabular} & $\begin{array}{l}\text { - Souza et al. }(2016)^{13} \\
\text { - Lins et al. }(2007)^{19} \\
\text { - Pedroso et al. }(2012)^{10} \\
\text { - Silva et al. }(2016)^{18}\end{array}$ \\
\hline & \begin{tabular}{|l} 
Número total de \\
AIHs (autorização \\
de internação \\
hospitalar) faturadas
\end{tabular} & AIHS & $\begin{array}{l}\text { Sistema de } \\
\text { Informação Hospitalar } \\
\text { Descentralizado - } \\
\text { SIHD }^{20}\end{array}$ & $\begin{array}{l}\text { - Silva et al. }(2017)^{17} \\
\text { - Silva et al. }(2016)^{18}\end{array}$ \\
\hline & $\begin{array}{l}\text { Número total de dias } \\
\text { de permanência SUS } \\
\text { (diárias) }\end{array}$ & PERM & \begin{tabular}{|l} 
Sistema de \\
Informação Hospitalar \\
Descentralizado - \\
SIHD
\end{tabular} & - Gonçalves et al. $(2007)^{21}$ \\
\hline & $\begin{array}{l}\text { Número total de } \\
\text { procedimentos } \\
\text { hospitalares de alta } \\
\text { complexidade SUS } \\
\text { realizados }\end{array}$ & AC & $\begin{array}{l}\text { Sistema de } \\
\text { Informação Hospitalar } \\
\text { Descentralizado - } \\
\text { SIHD }^{20}\end{array}$ & $\begin{array}{l}\text { - Souza et al. }(2016)^{13} \\
\text { - Lins et al. }(2007)^{19}\end{array}$ \\
\hline
\end{tabular}

Fonte: Elaborado pelos autores.

Utilizou-se o método da análise envoltória de dados por meio do software MDeap 2 para Windows. A análise foi realizada por intermédio de unidades comparáveis, ou seja, que desempenham as mesmas funções. O objetivo da comparação foi evidenciar as unidades ineficientes para a intervenção da gestão, no sentido do seu aperfeiçoamento ${ }^{22}$.

A análise parte do princípio de que existe um número de unidades decisórias (DMUs) que convertem insumos (inputs) em produtos (outputs). Assim, um hospital utiliza seus médicos, enfermeiros, leitos etc. como insumos para gerar produtos, tais como internações. Dessa forma, com os dados disponíveis para essas variáveis em vários hospitais, é possível avaliar a eficiência rela- tiva das diversas unidades hospitalares a partir de uma comparação entre os insumos e a geração de produtos. Outro aspecto importante é a diferença entre produtos e resultados. Embora o objetivo das unidades decisórias seja a obtenção de resultados, e não a mera geração de produtos, os primeiros são de mais difícil avaliação, uma vez que a quantidade de fatores externos que contribuem para a sua produção pode ser muito grande ${ }^{23}$.

O estudo foi de risco mínimo, pois foram utilizados somente os dados secundários de uma base que disponibiliza essas informações para o acesso ao público em geral (Departamento de Informática do SUS). Assim, a presente investigação não foi submetida ao Comitê de Ética em Pesquisa. 


\section{Resultados}

No mês de maio de 2017, o Brasil contava com 5.129 hospitais gerais com cadastro ativo no CNES, destes, somente 389 (7,6\%) possuem algum tipo de atividade de ensino, entre elas: unidade universitária (25), unidade de escola superior isolada (5), unidade auxiliar de ensino (238) ou hospital de ensino (121) - objeto desse estudo. Esses 389 hospitais correspondem a $28,1 \%$ do total de leitos de internação clínicos e cirúrgicos e a $48,3 \%$ dos leitos de Unidade de Terapia Intensiva.

A população desse estudo, após delimitação, constituiu-se de 29 hospitais pertencentes às regiões Centro-Oeste, Sul, Sudeste e Norte do Brasil (do total de 121 hospitais de ensino existentes no Brasil). A região Nordeste não possuiu hospital que se enquadrasse na delimitação estabelecida, no ano estudado, por isso não foi contemplada. Dos incluídos na amostra, conforme a estrutura da natureza jurídica, 14 pertencem à administração pública, quatro a entidades empresariais e 11 a entidades sem fins lucrativos. Nos modelos DEA, esses hospitais são denominados DMUs, sendo avaliados como eficientes ou ineficientes.

O Quadro 2 apresenta os escores de eficiência dos hospitais de ensino que compuseram a população deste estudo. Uma unidade (DMU) que obtenha o valor máximo da maximização da eficiência (1, por definição) é considerada "eficiente", e caso contrário é considerada "ineficiente". Observa-se que 12 hospitais estavam localizados na fronteira de eficiência, ou seja, foram identificados como eficientes na maximização dos outputs (produtos). Por sua vez, 17 ficaram abaixo dessa fronteira. Além disso, verifica-se que, desses 17 hospitais, a DMU 07 (Hospital São Paulo Hospital Universitário da UNIFESP), com escore de 0,50 , apresentou o pior resultado entre os ineficientes.

A Tabela 1 demonstra a relação de benchmarks para cada unidade considerada ineficiente, onde quanto mais alto o valor encontrado, mais forte a semelhança para o espelhamento. Percebe-se que a DMU 16 - Hospital Universitário Clemente de Faria - foi o hospital que mais vezes foi considerado benchmark de uma unidade ineficiente (12 vezes) e obteve o valor mais alto para a semelhança com a DMU 19 - Hospital Nossa Senhora da Conceição AS - $(4,15)$.

$\mathrm{Na}$ Tabela 2 observam-se os valores estimados das saídas (outputs) necessários para que cada DMU atingisse $100 \%$ de eficiência. Por exemplo, a DMU 01 - Hospital Universitário Maria
Aparecida Pedrossian - teve o valor faturado de $\mathrm{R} \$ 14.111 .100,00,9.858$ AIHs (autorização de internação hospitalar) processadas, 76.558 diárias aprovadas e 1.178 procedimentos de alta complexidade realizados. Nesse caso, para que o hospital atingisse $100 \%$ de eficiência, seria necessário aumentar o valor faturado para $\mathrm{R} \$ 18.228 .600,00$, processar $10.048 \mathrm{AIHs}$, aprovar 78.034 diárias e realizar 1.201 procedimentos de alta complexidade, de acordo com a sua quantidade de insumos fixos existentes.

A classificação da natureza jurídica dos hospitais foi dividida em três categorias: entidades sem fins lucrativos (11 hospitais), administração pública (14) e entidade empresarial (4). Nessa amostra foi possível identificar que os estabelecimentos com a personalidade jurídica entidade empresarial foram mais eficientes (média do escore de eficiência de 0,95 ), seguidos das entidades sem fins lucrativos $(0,93)$ e, por último, dos hospitais da administração pública $(0,91)$.

\section{Discussão}

O modelo data envelopment analysis é uma ferramenta matemática, não paramétrica, usada para mensurar a eficiência de unidades produtivas, chamadas decision making units - DMUs. O método utiliza a programação linear para calcular um índice de eficiência para cada DMU e gera uma fronteira de eficiência empírica, composta das unidades que apresentam as melhores práticas (benchmarks) específicas para a amostra pesquisada. As unidades da fronteira são classificadas como eficientes e as outras como ineficientes. O modelo DEA é apropriado para incorporar diversos inputs (insumos) e outputs (produtos). A eficiência de uma DMU é a razão entre sua própria produtividade e a produtividade da DMU mais eficiente no conjunto pesquisado ${ }^{24}$. Os resultados expostos, no entanto, necessitam de cautela ao serem analisados, pois a classificação como eficiente pode ser interpretada também como um indicador no balanço entre inputs e outputs, além de o valor máximo atingido pelo hospital não significar ausência de problemas na unidade, mas sim que conseguiram produzir mais resultados (outputs) com menos recursos (inputs), não sendo avaliado nesse caso a qualidade, devido à carência de dados secundários que abordem esse quesito, como informações sobre o controle de infecção e segurança do paciente.

No modelo DEA, a projeção espacial das unidades ineficientes na fronteira está delimitada por 
Quadro 2. Escores de eficiência relativa conforme a DEA.

\begin{tabular}{|c|c|c|c|c|c|}
\hline $\begin{array}{c}\mathrm{N}^{\circ} \\
\mathrm{DMU}\end{array}$ & Nome do Hospital & Natureza Jurídica & Região & Escore & Eficiente \\
\hline 01 & Universitário Maria Aparecida Pedrossian & Entidade empresarial & $\mathrm{CO}$ & 0,98 & Não \\
\hline 02 & Regional de Mato Grosso do Sul & Administração pública & $\mathrm{CO}$ & 0,96 & Não \\
\hline 03 & Materno Infantil de Brasília & Administração pública & $\mathrm{CO}$ & 0,89 & Não \\
\hline 04 & Hospital do Trabalhador & Administração pública & S & 1,00 & Sim \\
\hline 05 & Clínicas da UFMG & Entidade empresarial & SE & 1,00 & Sim \\
\hline 06 & Santa Marcelina & Entidade sem fins lucrativos & SE & 0,96 & Não \\
\hline 07 & Universitário da UNIFESP & Entidade sem fins lucrativos & SE & 0,50 & Não \\
\hline 08 & Guilherme Álvaro Santos & Administração pública & SE & 0,70 & Não \\
\hline 09 & Clínicas da UNICAMP de Campinas & Administração pública & SE & 0,93 & Não \\
\hline 10 & Conjunto Hospitalar Sorocaba & Administração pública & SE & 0,96 & Não \\
\hline 11 & Clínicas FAEPA Ribeirão Preto & Entidade sem fins lucrativos & SE & 0,95 & Não \\
\hline 12 & Clínicas Samuel Libânio Pouso Alegre & Entidade sem fins lucrativos & SE & 1,00 & Sim \\
\hline 13 & Clínicas de Uberlândia & Administração pública & SE & 0,85 & Não \\
\hline 14 & Metropolitano Odilon Behrens & Administração pública & SE & 0,96 & Não \\
\hline 15 & Clínicas da UFTM & Administração pública & SE & 0,83 & Não \\
\hline 16 & Universitário Clemente de Faria & Administração pública & SE & 1,00 & Sim \\
\hline 17 & Hospital Geral & Entidade sem fins lucrativos & S & 1,00 & Sim \\
\hline 18 & Irmandade Santa Casa de Misericórdia & Entidade sem fins lucrativos & $S$ & 1,00 & Sim \\
\hline 19 & Nossa Senhora da Conceição SA & Entidade empresarial & S & 0,83 & Não \\
\hline 20 & Hospital de Clínicas & Entidade empresarial & $\mathrm{S}$ & 1,00 & Sim \\
\hline 21 & Universitário de Santa Maria & Administração pública & $\mathrm{S}$ & 0,76 & Não \\
\hline 22 & São Vicente de Paulo & Entidade sem fins lucrativos & $\mathrm{S}$ & 1,00 & Sim \\
\hline 23 & Universitário São Francisco de Paula & Entidade sem fins lucrativos & S & 0,81 & Não \\
\hline 24 & São Lucas da PUCRS & Entidade sem fins lucrativos & $\mathrm{S}$ & 1,00 & Sim \\
\hline 25 & Clínicas Gaspar Viana & Administração pública & $\mathrm{N}$ & 1,00 & Sim \\
\hline 26 & Nossa Senhora da Conceição & Entidade sem fins lucrativos & $\mathrm{S}$ & 1,00 & Sim \\
\hline 27 & Universitário São Francisco na Providência & Entidade sem fins lucrativos & SE & 1,00 & Sim \\
\hline 28 & Clínicas de Botucatu & Administração pública & SE & 0,93 & Não \\
\hline 29 & Universitário Regional do Norte do Paraná & Administração pública & S & 0,96 & Não \\
\hline
\end{tabular}

Fonte: Elaborado pelos autores.

um conjunto de referência de unidades eficientes denominadas benchmark ${ }^{19}$. Para atingir a fronteira, os hospitais ineficientes podem estudar os valores absolutos das variáveis de seus benchmarks para que possíveis mudanças sejam operacionalizadas. Os dados de outro estudo ${ }^{13}$ mostraram que, quanto à eficiência total (CCR), os hospitais privados se mostraram mais eficientes que os públicos. Ainda nesse estudo, foi destacada uma limitação importante em relação à comparação entre hospitais públicos e privados (lucrativos e não lucrativos), visto que são estabelecimentos que operam sob lógicas diferentes. Os públicos têm um papel social a cumprir, enquanto os privados atuam sob a lógica do mercado, que é muito particular no caso dos serviços de saúde ${ }^{13}$. Mesmo identificada essa diferença na amostra, os autores entendem como importante essa comparação, uma vez que está em alta a discussão sobre privatização do setor de saúde. Argumentos que defendam ou critiquem essas posições são escassos na literatura atual e precisam ser mais explorados, a fim de subsidiar a decisão de gestores públicos.

Quando comparamos a natureza jurídica, é importante destacar que no caso de hospitais com administração pública, sob a ótica do financiamento, o custo com o pagamento dos servidores/funcionários não está diretamente relacionado ao recurso recebido mensalmente pela produção executada. Mesmo com essa vantagem em relação aos estabelecimentos de entidades empresariais e entidades sem fins lucrativos, os que possuem administração pública obtiveram na média do escore de eficiência o menor índice. 
Tabela 1. Benchmark para as ineficientes.

\begin{tabular}{lrrrrrrrrrrrr}
\hline DMU & $\mathbf{0 4}$ & $\mathbf{0 5}$ & $\mathbf{1 2}$ & $\mathbf{1 6}$ & $\mathbf{1 7}$ & $\mathbf{1 8}$ & $\mathbf{2 0}$ & $\mathbf{2 2}$ & $\mathbf{2 4}$ & $\mathbf{2 5}$ & $\mathbf{2 6}$ & $\mathbf{2 7}$ \\
\hline 01 & - & - & 0,12 & 0,43 & - & - & - & - & 0,05 & 0,46 & - & - \\
02 & - & - & 0,15 & 0,89 & - & - & - & - & 0,16 & 0,45 & - & - \\
03 & 0,39 & - & 0,17 & 0,05 & 0,68 & - & - & - & - & - & - & - \\
06 & - & - & - & - & - & - & - & 0,31 & 1,01 & 0,53 & - & - \\
07 & - & - & - & 0,79 & - & - & - & - & 1,84 & 0,05 & - & - \\
08 & - & - & 0,38 & 0,57 & - & - & - & - & - & 0,25 & - & - \\
09 & 0,11 & - & - & - & - & - & - & 0,53 & 1,01 & - & - & - \\
10 & - & - & - & 1,18 & - & - & - & - & 0,19 & 0,32 & - & - \\
11 & 0,42 & 0,17 & - & - & - & - & - & 0,27 & 1,45 & - & - & - \\
13 & - & - & - & 0,19 & - & - & - & 0,25 & 1,01 & 0,15 & - & - \\
14 & - & - & - & 1,81 & - & - & - & 0,35 & - & - & 0,11 & - \\
15 & 0,04 & - & - & - & - & - & - & - & 0,74 & 0,18 & - & - \\
19 & - & - & - & 4,15 & - & - & - & - & - & - & 2,08 & - \\
21 & - & - & - & 0,12 & - & - & 0,22 & - & 0,17 & 0,59 & - & - \\
23 & - & - & - & 0,82 & - & - & - & - & - & 0,06 & 0,2 & - \\
28 & 0,36 & - & - & - & - & - & - & - & 1,13 & 0,01 & - & - \\
29 & - & - & - & 0,33 & 0,49 & - & - & - & 0,37 & - & - & - \\
Qtd. & 6 & 1 & 4 & 12 & 1 & 0 & 1 & 6 & 11 & 10 & 3 & 1 \\
\hline
\end{tabular}

Fonte: Elaborado pelos autores.

Tabela 2. Metas de produção para as DMUs ineficientes.

\begin{tabular}{|c|c|c|c|c|c|c|c|c|}
\hline \multirow{3}{*}{ DMU } & \multicolumn{8}{|c|}{ OUTPUT (produtos) } \\
\hline & \multicolumn{2}{|c|}{ VALOR } & \multicolumn{2}{|c|}{ AIHS } & \multicolumn{2}{|c|}{ PERM } & \multicolumn{2}{|c|}{$\mathrm{AC}$} \\
\hline & Realizado & Meta & Realizado & Meta & Realizado & Meta & Realizado & Meta \\
\hline 01 & $\mathrm{R} \$ 14.111 .100$ & $\mathrm{R} \$ 18.228 .600$ & 9.858 & 10.048 & 76.558 & 78.034 & 1.178 & 1.201 \\
\hline 02 & $\mathrm{R} \$ 25.684 .300$ & $\mathrm{R} \$ 26.635 .300$ & 15.317 & 15.884 & 112.668 & 116.839 & 1.365 & 1.708 \\
\hline 03 & $\mathrm{R} \$ 17.629 .200$ & $\mathrm{R} \$ 31.170 .100$ & 16.838 & 18.839 & 76.109 & 85.152 & 12 & 2.459 \\
\hline 06 & $\mathrm{R} \$ 72.883 .200$ & $\mathrm{R} \$ 76.061 .900$ & 27.957 & 29.176 & 192.374 & 200.764 & 5.427 & 7.135 \\
\hline 07 & $\mathrm{R} \$ 40.899 .600$ & $\mathrm{R} \$ 83.854 .200$ & 18.323 & 37.197 & 124.852 & 248.001 & 3.667 & 7.284 \\
\hline 08 & $\mathrm{R} \$ 11.121 .400$ & $\mathrm{R} \$ 17.101 .300$ & 8.637 & 12.403 & 57.083 & 81.972 & 436 & 1.412 \\
\hline 09 & $\mathrm{R} \$ 76.503 .800$ & $\mathrm{R} \$ 82.182 .800$ & 29.799 & 32.011 & 178.978 & 192.264 & 5.746 & 8.294 \\
\hline 10 & $\mathrm{R} \$ 22.942 .500$ & $\mathrm{R} \$ 24.606 .100$ & 12.891 & 15.500 & 109.221 & 114.264 & 1.217 & 1.273 \\
\hline 11 & R\$ 99.941.700 & $\mathrm{R} \$ 105.425 .000$ & 39.868 & 42.056 & 233.070 & 247.788 & 9.086 & 9.585 \\
\hline 13 & $\mathrm{R} \$ 54.831 .200$ & $\mathrm{R} \$ 64.291 .200$ & 21.408 & 25.782 & 143.886 & 168.711 & 4.378 & 6.121 \\
\hline 14 & $\mathrm{R} \$ 40.530 .100$ & $\mathrm{R} \$ 42.071 .600$ & 24.051 & 25.227 & 155.031 & 160.927 & 799 & 3.199 \\
\hline 15 & $\mathrm{R} \$ 29.956 .300$ & $\mathrm{R} \$ 36.198 .700$ & 11.991 & 14.490 & 81.178 & 98.094 & 1.831 & 3.214 \\
\hline 19 & $\mathrm{R} \$ 60.871 .000$ & $\mathrm{R} \$ 95.456 .400$ & 35.335 & 63.184 & 334.853 & 402.519 & 4.517 & 6.178 \\
\hline 21 & $\mathrm{R} \$ 17.344 .400$ & $\mathrm{R} \$ 38.847 .900$ & 12.235 & 16.102 & 97.494 & 128.306 & 2.423 & 3.189 \\
\hline 23 & $\mathrm{R} \$ 10.923 .600$ & $\mathrm{R} \$ 13.758 .000$ & 6.768 & 9.964 & 54.487 & 67.061 & 323 & 697 \\
\hline 28 & $\mathrm{R} \$ 54.831 .200$ & $\mathrm{R} \$ 58.691 .400$ & 23.577 & 25.237 & 135.712 & 145.266 & 4.551 & 5.139 \\
\hline 29 & $\mathrm{R} \$ 27.699 .900$ & $\mathrm{R} \$ 28.839 .000$ & 13.758 & 15.078 & 87.687 & 91.293 & 1.833 & 2.326 \\
\hline
\end{tabular}

Fonte: Elaborado pelos autores. 
Diferentemente de outros estudos que utilizaram a metodologia DEA na área de avaliação em saúde no país, este se restringiu a hospitais de ensino que possuíam características semelhantes de capacidade instalada, e não a hospitais ou regiões como um todo. Buscou-se, assim, garantir a realização de comparações em entidades intrinsecamente de maior homogeneidade. Para tal, foram utilizados indicadores clássicos já utilizados em estudos semelhantes, como diárias de permanência e volume de produção, relacionando-os com a capacidade instalada de leitos e horas de profissionais.

Um desafio importante é como considerar o contexto de operação dos hospitais em abordagens quantitativas. Tratando-se de hospitais do SUS, elementos como organização ou desorganização do sistema, contexto sociopolítico, financiamento, tempo de uso e estado de conservação dos hospitais são importantes e podem interferir no desempenho e até explicar determinados resultados encontrados ${ }^{13}$.

Reunindo os resultados alcançados pelo estudo, foi possível evidenciar que $59 \%$ dos hospitais de ensino que compuseram a amostra têm potencial em insumos já existentes para ampliar a sua produção, por meio do aumento da oferta de serviços à população usuária do SUS. A ferramenta DEA, além de estabelecer as metas possíveis para a produção dos ineficientes, indica o benchmark com os hospitais mais fortes para o espelhamento. Dessa forma, é possível uma aproximação entre esses estabelecimentos, buscando alternativas para a ampliação da utilização dos recursos existentes e a troca de experiências de sucesso entre eles.

Embora os hospitais de ensino sejam unidades com mais recursos tecnológicos que a média dos demais serviços do SUS, constata-se que ainda realizam uma grande proporção de atendimentos de média complexidade, tanto de internação como ambulatoriais, que poderiam ser encaminhados para outros serviços do sistema. A realização de procedimentos de baixa complexidade nos hospitais de ensino apresenta vários inconvenientes, entre os quais: utilização de um serviço de alta capacidade instalada, com pessoal especializado, portanto com atendimentos em média mais custosos para procedimentos simples que seriam melhor atendidos em outros níveis de atenção; os pacientes, na busca do atendimento continuado nos hospitais de ensino para problemas básicos de saúde, são forçados a deslocamentos frequentes para fora de seu município, pois esses hospitais são habitualmente localizados em municípios maiores, polos de sua região; e, finalmente, os atendimentos de menor complexidade bloqueiam as agendas para os procedimentos de maior complexidade, provocando o surgimento de filas de espera e demanda reprimida, o que prejudica os pacientes e atrasa os atendimentos ${ }^{4}$.

As considerações realizadas sobre a eficiência técnica nos hospitais de ensino contribuem para as discussões que emergem acerca da utilização de recursos existentes e metodologias que auxiliem na identificação de alternativas para a ampliação do acesso da população à saúde em todos os níveis de atenção, além de inevitavelmente se refletir no financiamento do sistema de saúde. Os resultados deste estudo poderão subsidiar a implementação de mecanismos mais ampliados de controle e a avaliação dos serviços de saúde, além de fornecer aos gestores locais informações relevantes a respeito da situação atual da produção dos grandes hospitais de ensino do Brasil, independentemente da natureza jurídica.

\section{Considerações finais}

A dimensão de eficiência hoje se encontra no topo das agendas dos gestores de saúde, e a técnica DEA possibilita a construção de consensos e a consideração de prioridades, com a validade das aplicações podendo ser enriquecida pela introdução de julgamento de valor e da interação com especialistas. O presente estudo permitiu demonstrar que a DEA tem capacidade de se tornar uma importante ferramenta para avaliar os serviços e auxiliar na tomada de decisão em saúde. Para tanto, um caminho factível está na aproximação disciplinar entre a epidemiologia de serviços de saúde e a pesquisa operacional ${ }^{25}$.

A utilização da metodologia demonstra as potencialidades da DEA para avaliação de programas governamentais e políticas públicas, contratualização de metas, priorização de ações corretivas etc., na medida em que identifica, por variável, as folgas ou níveis de esforços necessários para o alcance de patamares mais eficientes de gestão. A técnica também possui características positivas de neutralidade e acolhe participações possíveis e desejáveis de gestores e de outros agentes na avaliação. Essa participação é desejável, dada a estrutura complexa de governança adotada pelo SUS. Além disso, permite a qualificação e a determinação de caminhos possíveis para a fronteira de eficiência para fins de monitoramento dos objetivos propostos ${ }^{10}$.

A realidade atual do país não permite que os gestores públicos vislumbrem aumento de gastos 
para o setor, tornando cada vez mais necessária a avaliação baseada nos recursos disponíveis com o objetivo de maximizar os resultados dos serviços prestados tanto em regiões mais desenvolvidas como em localidades que carecem de infraestrutura adequada. O Brasil, por ser um país de vastas extensões geográficas, precisa ajustar algumas falhas sistêmicas organizacionais, isto é, convencionar as políticas direcionadas para a área de saúde e as equipes responsáveis pela condução das mesmas, para que estas não permaneçam inoperantes devido ao abuso de burocracia e tenham força para combater as desigualdades sociais, tanto no âmbito federal quanto nos âmbitos estadual e municipal ${ }^{1}$.

Uma dificuldade encontrada na avaliação da eficiência técnica de hospitais é a seleção de insumos e produtos hospitalares para a avaliação. Medidas inadequadas podem causar vieses e inconsistências de resultados da análise de eficiência. A literatura existente sugere como produto hospitalar o número de internações, o número de pacientes-dia ou de altas hospitalares, na maioria das vezes ignorando a gravidade das condições de saúde do paciente e a qualidade dos serviços prestados. Em muitos estudos sobre desempenho produtivo em hospitais essa limitação é expressa como ausência de dados disponíveis para consulta ${ }^{5}$.

A principal limitação deste estudo está relacionada à confiabilidade dos dados disponibilizados pelo DATASUS, uma vez que dependem da atualização de cada hospital, e na maioria das vezes não há fiscalização ou auditoria para a validação dos mesmos. Dessa forma, o conjunto de variáveis coletadas poderá não refletir plenamente a realidade de eficiência em hospitais que por ventura tiveram falhas na alimentação de seus dados. Além disso, é possível registrar a limitação quanto à disponibilidade de dados que reflitam a qualidade dos serviços prestados, sendo esses importantes para uma avaliação mais minuciosa acerca do tema produção, tanto hospitalar quanto ambulatorial. Investigações mais aprofundadas sobre o tema eficiência técnica em hospitais de ensino, com outras dimensões importantes como regulação, financiamento, incentivos, modelos de contratualização e perfil epidemiológico da região, ainda podem ser exploradas.

Os resultados desta pesquisa sugerem que a DEA tem potencial para avaliação de eficiência técnica no âmbito hospitalar quando avaliada a capacidade operacional do estabelecimento. Considerando tratar-se de uma amostra em nível nacional, só foram consideradas variáveis com fonte em dados secundários públicos, onde infelizmente há a carência de informações relacionadas à qualidade assistencial no universo do SUS. Cada estudo tem suas especificidades e os resultados se restringem ao grupo analisado e contexto aplicado, não possibilitando extrapolar inferências sobre outras categorias.

\section{Colaboradores}

A Garmatz realizou a escrita original, elaborou o banco de dados e o analisou. SA Sirena realizou todas as revisões e auxiliou na metodologia. GBB Vieira realizou a revisão das variáveis utilizadas e a aplicação da metodologia da análise envoltória de dados. 


\section{Referências}

1. Medeiros RVV, Marcolino VA. A eficiência dos municípios do Rio de Janeiro no setor de saúde: Uma análise através da DEA e regressão logística. Meta: Avaliação, 2018; 10(28):183-210.

2. Faria TC. Tecnologia de gestão hospitalar: proposta e aplicação em SC [monografia]. Florianópolis: Universidade Federal de Santa Catarina; 2016.

3. Fonseca PC, Ferreira MAM. Investigação dos níveis de eficiência na utilização de recursos no setor de saúde: uma análise das microrregiões de Minas Gerais. Saude Soc 2009; 18(2):199-213.

4. Barata LRB, Mendes JDV, Bittar OJNV. Hospitais de ensino e o Sistema Único de Saúde. Rev adm saude 2010; 12(46):7-14

5. Wolff, LDG. Um modelo para avaliar o impacto do ambiente operacional na produtividade de hospitais brasileiros [tese]. Florianópolis: Universidade Federal de Santa Catarina; 2005.

6. Vecina Neto G, Malik AM. Tendências na assistência hospitalar. Cien Saude Colet 2007; 12(4):825-839.

7. Giancotti M, Rotundo G, Pipitone V, Mauro M. Efficiency and optimal size of Italian public hospitals: results from data envelopment analysis. Epidemiology Biostatistics and Public Health, 2018; 5(15):e129291-e12929-12.

8. Scaratti D, Calvo MCM. Indicador sintético para avaliar a qualidade da gestão municipal da atenção básica à saúde. Rev Saude Publ 2012; 46(3):446-455.

9. Fitzsimmons JA, Fitzsimmons MJ. Administração de serviços: operações, estratégia e tecnologia da informação. Porto Alegre: AMGH; 2014.

10. Pedroso MM, Calmon PCDP, Bandeira LF, Lucena RAV. Eficiência relativa da política nacional de procedimentos cirúrgicos eletivos de média complexidade. Rev adm contemp 2012; 16(2):237-252.

11. Lobo MSC. Aplicação da análise envoltória de dados (DEA) para apoio às políticas públicas de saúde: o caso dos hospitais de ensino [tese]. Rio de Janeiro: Universidade Federal do Rio de Janeiro; 2010.

12. Senra LFAC, Nanci LC, Mello JCCBS, Meza LA. Estudo sobre métodos de seleção de variáveis em DEA. Pesq Oper 2007; 27(2):191-207.

13. Souza PC, Scatena JHG, Kehrig RT. Aplicação da análise envoltória de dados para avaliar a eficiência de hospitais do SUS em Mato Grosso. Physis 2016; 26(1):289-308

14. Calvo MCM. Hospitais públicos e privados no Sistema Único de Saúde do Brasil: o mito da eficiência privada no estado de Mato Grosso em 1998 [tese]. Florianópolis: Universidade Federal de Santa Catarina; 2002.

15. Bandeira DL. Análise da eficiência relativa de departamentos acadêmicos - o caso da UFRGS [dissertação]. Porto Alegre: Universidade Federal do Rio Grande do Sul; 2000.

16. Departamento de Informática do SUS. Cadastro Nacional de Estabelecimentos de Saúde [site da Internet]. [acessado 2018 maio 14]. Disponível em: http:// datasus.saude.gov.br/informacoes-de-saude/servi$\cos 2 /$ transferencia-de-arquivos.
17. Silva BN, Costa MAS, Abbas K, Galdamez EVC. Eficiência hospitalar das regiões brasileiras: um estudo por meio da análise envoltória de dados. RGSS 2017; 6(1):76-91

18. Silva MZ, Moreth BR, Schuster HA. Avaliação da eficiência hospitalar por meio da análise envoltória de dados. RGSS 2016; 5(2):100-114.

19. Lins ME, Lobo MSC, Silva ACM, Fiszman R, Ribeiro VJP. O uso da análise envoltória de dados (DEA) para avaliação de hospitais universitários brasileiros. Cienc Saude Colet 2007; 12(4):985-998.

20. Departamento de Informática do SUS. Sistema de Informação Hospitalar [site da Internet]. [acessado 2018 jul 11]. Disponível em: http://datasus.saude.gov. br/informacoes-de-saude/servicos2/transferencia-de -arquivos.

21. Gonçalves AC, Noronha CP, Lins MPE, Almeida RMVR. Análise envoltória de dados na avaliação de hospitais públicos nas capitais brasileiras. Rev Saude Publ 2007; 41(3):427-435.

22. Andrade EL. Introdução à pesquisa operacional: métodos e modelos para análise de decisões. Rio de Janeiro: LTC; 2015.

23. Boueri R, Rocha F, Rodopoulo F, organizadores. Avaliação da qualidade do gasto público e mensuração da eficiência. Brasília: Secretaria do Tesouro Nacional; 2015.

24. Silva RM, Vieira GBB, Senna ETP, Senna LAS. A aplicação da data envelopment analysis (DEA) para análise da produtividade na atividade de picking em um centro de distribuição. Rev Eng Tec 2016; 8(1):35-49.

25. Lobo MSC, Lins MPE. Avaliação da eficiência dos serviços de saúde por meio da análise envoltória de dados. Cad Saude Publica 2011; 19(1):93-102.

Artigo apresentado em 12/06/2019

Aprovado em 18/12/2019

Versão final apresentada em 20/12/2019

Editores-chefes: Romeu Gomes, Antônio Augusto Moura da Silva 
